\title{
Effect of Intralesional Triamcinolone Injection in Patients of Esophageal Stricture Post Acid Ingestion
}

\author{
Dr. Sushil Narang ${ }^{1}$, Dr. Sandeep Kansal ${ }^{2}$, Dr. Sonny Bherwani ${ }^{3}$, \\ Dr. Sushruth Shetty ${ }^{4}$, Dr. Kaushal Y Vyas ${ }^{5}$, \\ ${ }^{l}$ DM Gastroenterology, Assistant Professor, VS Hospital, Smt. NHL Municipal Medical College, Ahmedabad, \\ Gujarat \\ ${ }^{2}$ Associate Professor, Surgery, Govt. Medical College, Surat \\ ${ }^{3}$ DM Gastroenterology Resident, VS Hospital, Smt. NHL Municipal Medical College, Ahmedabad, Gujarat \\ ${ }^{4}$ MCH Surgery Resident, VS Hospital, Smt. NHL Municipal Medical College, Ahmedabad, Gujarat \\ ${ }^{5}$ Professor and Head, DM Gastroenterology, VS Hospital, Smt. NHL Municipal Medical College, Ahmedabad, \\ Gujarat
}

\begin{abstract}
:
Introduction:Up to one third of corrosive ingestion patients will develop esophageal stricture after initial recovery. The primary treatment of esophageal strictures secondary to caustic ingestion is frequent dilation. Refractory cases may respond to intralesional streroids. We aimed to study the effect of intralesional triamcinolone injection in patients who present to our hospital with esophageal strictures post acid ingestion.

Methodology: After obtaining approval of the ethics committee, a prospective study was conducted in the Department of Gastroenterology, Smt. NHL Municipal Medical College, Ahmedabad, Gujarat from Sept 2015 to Aug 2016. We included all patients who presented to the Emergency Ward with the history of acid ingestion and were refractory to conventional endoscopic balloon dilation with Savary-Gilliard balloon dilator. Triamcinolone acetonide $(40 \mathrm{mg} / \mathrm{mL}$, diluted 1:1 with saline solution) was injected in four quadrants using a 23 gauge, $5 \mathrm{~mm}$ long sclerotherapy needle in aliquots of $0.5 \mathrm{~mL}$ at the proximal margin of the stricture and into the strictured segment. Number of dilations needed before and after steroid injection and dysphagia score was noted for each patient.
\end{abstract}

Results: For 64 patients, mean age of the patients were 22.74 years and 42 were males. Mean number of dilations needed before streroid injection were significantly more than after steroid injection $(p<0.0001)$. Similarly, proportion of patients who could achieve atleast $15 \mathrm{~mm}$ dilation was significantly higher after steroid injection as compared to before streroid injection ( $p<0.0001$ ). Mean dysphagia score also improved signifiantly from 2.64 to 0.81 before and after steroid injection respectively $(p<0.0001)$.

Conclusions: Findings of our study support the use of intralesional triamcinolone injections for patients who are refractory to repeated dilations for esophageal stricture after acid ingestion.

Keywords:dysphagia, stricture, esophagus, intralesional

\section{Introduction}

Gastroenterologists frequently encounter esophageal strictures in their clinical practice. Benign strictures are caused by a variety of reasons of which ingestion of corrosive fluids is very common. Up to one third of corrosive ingestion patients will develop esophageal stricture after initial recovery. Stricture formation presents most commonly at 2 months after injury but can occur at any time from 2 weeks to many years after the initial injury ${ }^{1}$.Esophageal strictures develop in areas of anatomic narrowing (thoracic inlet, gastroesophageal junction, or point of compression where the left bronchus crosses the esophagus), where contact with the caustic agent is more prolonged. Strictures occur only with full-thickness esophageal necrosis and prevalence of stricture formation varies from $10 \%$ to $50 \%^{2}$.

The primary treatment of esophageal strictures secondary to caustic ingestion is frequent dilation. Some patients with benign esophageal strictures do not achieve acceptable symptom relief despite an intensive dilation schedule and gastroesophageal reflux therapy. Previous studies have reported better clinical outcomes in patients with esophageal strictures who are treated with intralesional steroid injections after dilation ${ }^{3}$.

We aimed to study the effect of intralesional triamcinolone injection in patients who present to our hospital with esophageal strictures post acid ingestion. 


\section{Study Setting and Design}

\section{Methodology}

A prospective study was designed to be conducted in the Department of Gastroenterology, Smt. NHL Municipal Medical College, Ahmedabad, Gujarat from Sept 2015 to Aug 2016. After obtaining approval of the institutional ethics committee, we included all patients who presented to the Emergency Ward with the history of acid ingestion and was later admitted to indoor ward for benign esophageal stricture. Only those patients who were refractory to conventional endoscopic balloon dilation were included in the study. Refractory strictures were defined as anatomic restriction due to fibrosis requiring more than three sessions of dilatation to maintain lumen of at least $14 \mathrm{~mm}$ or inability to maintain a satisfactory luminal diameter for 4 weeks once the target diameter of $14 \mathrm{~mm}$ had been achieved. We excluded patients who had esophageal strictures due to reasons other than acid ingestion, a complete stricture or with other major comorbid conditions. Informed written consent was obtained from each patient.

\section{Data Collection and Analysis}

At the time of enrollment for the study demographic and relevant historical information of the patient was collected. During the course of admission, clinical information of the patient like nature of acid ingestion, number of stricture, site and size of strictures was collected. In our hospital we use Savary-Gilliard balloon dilator. Most subsequent dilation procedures were scheduled in 2 weeks until endoscopic resolution of strictures and improvement in patient's symptoms, or earlier if patients returned with further dysphagia. When started on intralesional steroids, dilations were combined with triamcinolone acetonide $(40 \mathrm{mg} / \mathrm{mL}$, diluted 1:1 with saline solution) injections in four quadrants using a 23 gauge, $5 \mathrm{~mm}$ long sclerotherapy needle in aliquots of $0.5 \mathrm{mLat}$ the proximal margin of the stricture and into the strictured segment, given every 2 weeks for 2 months or more.During the followup for next 1 year, number of dilations needed by the patient before and after triancinolone injection were noted for each patient. We also noted how many patients could achieve a dilation of $15 \mathrm{~mm}$ or more. Along the course of the treatment we also calculated the dysphagia score for each patient. At each visit, patients were examined to assess the severity of dysphagia using the dysphagia score, which was graded on a scale of 0 to 4 (grade 0 , no dysphagia; grade 1, dysphagia with ingestion of solid food; grade 2, dysphagia to semisolid food, grade 3, dysphagia to liquids; grade 4, aphagia). After each dilation patients were observed for 4 hours with specific attention to the occur- rence of chest pain, abdominal pain, and difficulty in breathing and hemodynamic status. Patients were discharged home the same day with instructions to immediately report the development of fever, chest pain, or shortness of breath.Data was collected, tabulated and entered in Microsoft excel sheet. This data were imported in SPSS software for generating descriptive analysis and other statistical analysis were conducted as appropriate.

\section{Results}

During the study period we enrolled 67 patients, of which 3 were lost to followup. As a result a total of 64 patients were included in the final analysis of the study. Mean age of the patients were 22.74 years and 42 were males. (Table 1) Most common reason of acid ingestion was suicidal $(n=44)$, followed by accidental $(n=16) .39$ patients had one stricture and 20 had two strictures. Majority of the strictures were in the proximal esophagus $(\mathrm{n}=39)$. Mean number of dilations needed before streroid injection were significantly more than after steroid injection $(\mathrm{p}<0.0001)$. (Table 2) Similarly, proportion of patients who could achieve atleast $15 \mathrm{~mm}$ dilation was significantly higher after steroid injection as compared to before streroid injection $(\mathrm{p}<0.0001)$. Mean dysphagia score also improved signifiantly from 2.64 to 0.81 before and after steroid injection respectively $(\mathrm{p}<0.0001)$. (Table 3$)$ No major complications were reported.

\section{Discussion}

Very scare literature is available which can describe the experience of using intralesional steroid injections for refractory esophageal strictures. Early dilation is not recommended due to the associated high incidence of perforation and associated morbidity. Most clinicians wait three to six weeks after the initial injury before attempting dilation ${ }^{4}$.Perforation occurs in approximately $0.5 \%$ of procedures ${ }^{5}$. Some patients with benign esophageal strictures do not achieve acceptable symptom relief despite an intensive dilation schedule and gastroesophageal reflux therapy. These patients are often poor surgical candidates. In addition, for those who are surgical candidates, successful antireflux surgery for peptic strictures depends upon adequate preoperative esophageal dilation, which may be difficult to accomplish safely. The injection of corticosteroids into the stricture may reduce stricture recurrence following dilation.Our findings indicate that there is an overall improvement in the clinical picture of the patient. In our experience, after intralesional steroids, there was decrease in the number of dilations required by the patient and the mean dysphagia score and an increase in the number of patients who could achieve $15 \mathrm{~mm}$ of dilation. Similar resutls were seen in a study by Kochhar and Makharia, who reported a decrease in dysphagia score from 2.34 to $0.65^{6}$. 
In most studies triamcinolone acetate or acetonide in concentrations of $10 \mathrm{mg} / \mathrm{mL}$ has been used for intralesional injection, although Gandhi et $\mathrm{al}^{7}$ used a concentration of $40 \mathrm{mg} / \mathrm{mL}$, which is similar to ours. The volume per injection has also varied in the literature from $0.6 \mathrm{~mL}$ to $2.8 \mathrm{~mL}$. The mechanism of action is not well understood, but corticosteroids may impede collagen deposition and enhance its breakdown locally, thereby reducing scar formation ${ }^{8}$. A sham-controlled trial involving 30 patients with a peptic stricture and recurrent dysphagia found a significant reduction in the need for repeat dilation (13 versus $60 \%$ ) one year after the procedure in patients who received steroid injection ${ }^{9}$. However, a benefit of steroid injection was not demonstrated in a sham-controlled trial involving 60 patients with cervical anastomotic strictures following esophagectomy ${ }^{10}$. Intralesional steroids have also been combined with systemic corticosteroids ${ }^{11}$. Reports of succesfully using intralesional steroids to treat esophageal strictures go as back as $1950 \mathrm{~s}^{12}$. Animal models have also shown higher treatment rates with intralesional steroids ${ }^{13}$. Different case series have reported different response to corticosteroid injections, with some patients failing to respond even after multiple injections. This could be in part because of improper location of injection. Bhutani et al have suggested the use of an endoscopic ultrasound catheter probe to guide the injection into the thickest portion of the stricture ${ }^{14}$.

This study adds to the growing evidence of the efficacy of triamcinolone intralesional injections in benign refractory esophageal strictures with encouraging results in favour of triamcinolone injection use.

\section{Conclusions}

Our research findings support the use of intralesional triamcinolone injections for patients who are refractory to repeated dilations for esophageal stricture after acid ingestion. Moreover, our intervention did not cause any major complications in our patients. The use of intralesional triamcinolone injections in esophageal strictures due to other etiologies need to be explored further.

Table 1.Baseline Characteristics of Patients with Corrosive Esophageal Stricture

\begin{tabular}{|l|l|}
\hline Total number of patients & 64 \\
\hline Mean age & $\begin{array}{l}24.74 \pm 7.21 \text { years (range 14-58 } \\
\text { years) }\end{array}$ \\
\hline Males & 42 \\
\hline Nature of ingestion & 16 \\
\hline Accidental & 44 \\
\hline Suicidal & 04 \\
\hline Homicidal & 39 \\
\hline Number of strictures & 20 \\
\hline One & 5 \\
\hline Two & $1.4 \pm 0.3$ \\
\hline Three & 39 \\
\hline Mean & 15 \\
\hline Site of strictures & 10 \\
\hline Proximal third & 44 \\
\hline Middle third & 20 \\
\hline Distal third & \\
\hline Length of strictures & \\
\hline Less than 3 cms & \\
\hline More than 3 cms &
\end{tabular}

Table 2. Effect of intralesional triamcinolone injection on esophageal strictures

\begin{tabular}{|l|l|l|l|}
\hline Variable & Before injection & After injection & p value \\
\hline Number of dilations & & & \\
\hline Mean & 3.84 & 1.52 & $<0.0001$ \\
\hline SD & 3.21 & 1.18 & \\
\hline Number of patients that could be dilated to $15 \mathrm{~mm}$ & 15 & 49 & $<0.0001$ (one-tailed) \\
\hline
\end{tabular}

Table 3. Change in Mean Dysphagia Score in Patients

\begin{tabular}{|l|l|}
\hline Level of intervention & Mean Dysphagia Score \\
\hline Before dilation & $3.81(0.82)$ \\
\hline Before triamcinolone injection & $2.64(0.43)$ \\
\hline After triamcinolone injection & $0.81(0.70)$ \\
\hline
\end{tabular}




\section{References}

[1] Zargar SA, Kochlar R, Nagi B, et al: Ingestion of corrosive alkalis. Spectrum of injury to upper gastrointestinal tract and natural history. Am J Gastroenterol 1992; 87: pp. 337-341

[2] Lee M, Kubik CM, Polha- mus CD, Brady CE 3rd, Kadakia SC. Preliminary experience with endoscopic intralesional steroid injection therapy for refrac- tory upper gastrointestinal strictures. Gastrointest Endosc 1995; 41: 598-601

[3] Pereira-Lima JC, Bonotto ML, Hahn GD, Watte G, Lopes CV, dos Santos CE, Teixeira CR. A prospective randomized trial of intralesional triamcinolone injections after endoscopic dilation for complex esophagogastric anastomotic strictures. Surgical endoscopy. 2015 May 1;29(5):1156-60

[4] Keh SM, Onyekwelu N, McManus K, McGuigan J. Corrosive injury to upper gastrointestinal tract: Still a major surgical dilemma. World J Gastroenterol 2006; 12:5223.

[5] Temiz A, Oguzkurt P, Ezer SS, et al. Long-term management of corrosive esophageal stricture with balloon dilation in children. Surg Endosc 2010; 24:2287.

[6] Kochhar R, Makharia G K. Usefulness of intralesional triamcinolone in treatment of benign esophageal strictures. Gastrointest Endosc 2002; 56: 829-34.

[7] Gandhi RP, Cooper A, Barlow B. Successful management of esophageal strictures without resection or replacement. J Pediatr Surg 1989;24:745-50.

[8] Russell SB, Trupin JS, Myers JC, et al. Differential glucocorticoid regulation of collagen mRNAs in human dermal fibroblasts. Keloid-derived and fetal fibroblasts are refractory to down-regulation. J Biol Chem 1989; 264:13730.

[9] Ramage JI Jr, Rumalla A, Baron TH, et al. A prospective, randomized, double-blind, placebo-controlled trial of endoscopic steroid injection therapy for recalcitrant esophageal peptic strictures. Am J Gastroenterol 2005; 100:2419.

[10] Hirdes MM, van Hooft JE, Koornstra JJ, et al. Endoscopic corticosteroid injections do not reduce dysphagia after endoscopic dilation therapy in patients with benign esophagogastric anastomotic strictures. Clin Gastroenterol Hepatol 2013; 11:795.

[11] Morikawa N, Honna T, Kuroda T, et al. High dose intravenous methylprednisolone resolves esophageal stricture resistant to balloon dilatation with intralesional injection of dexamethasone. Pediatr Surg Int 2008; 24:1161.

[12] Holder TM, Ashcraft KW, Leape L. The treatment of patients with esophageal strictures by local steroid injections. Journal of pediatric surgery. 1969 Dec 1;4(6):646-53

[13] Ashcraft KW, Holder TM. The expeimental treatment of esophageal strictures by intralesional steroid injections. Journal of Thoracic and Cardiovascular Surgery. 1969 Nov 1;58(5).

[14] Bhutani MS, Usman N, Shenoy V, Qarqash A, Singh A, Bardec J, et al. Endoscopic ultrasound miniprobe-guided steroid injection for treatment of refractory esophageal strictures. Endoscopy 1997;29:757-9. 